\title{
A Real-Time Implementable NMPC Output Feedback for a Diesel Engine Air Path
}

\author{
A. Murilo ${ }^{1 *}$, M. Alamir ${ }^{2}$ and P. Ortner ${ }^{3}$ \\ 1 GIPSA-Lab, Control Systems Dept., University of Grenoble, Domaine Universitaire, 38400 Saint Martin d'Hères - France \\ 2 CNRS/Gipsa-Lab, Control Systems Dept., University of Grenoble, Domaine Universitaire, 38400 Saint Martin d'Hères - France \\ 3 Institute for Design and Control of Mechatronical Systems, JKU Linz, Altenberger Straße 69 1-4040, Linz, Austria \\ e-mail: andre.murilo@ufpe.br - mazen.alamir@gipsa-lab.grenoble-inp.fr - peter_ortner@gmx.net \\ * Corresponding author
}

Résumé - Commande prédictive non linéaire avec retour de sortie implémentable en tempsréel pour un circuit d'air d'un moteur Diesel - Dans cet article, une validation expérimentale d'un schéma de commande prédictive non linéaire (CPNL) paramétrique avec un observateur à horizon glissant a été proposé pour le circuit d'air d'un moteur Diesel. La stratégie de commande basée sur l'approche paramétrique à faible dimension s'est montrée implémentable en temps réel et peut être utilisée aussi en tant qu'une solution du type boîte noire indépendamment de la structure du modèle non linéaire.

Abstract - A Real-Time Implementable NMPC Output Feedback for a Diesel Engine Air Path - In this paper, an experimental validation of a parameterized Nonlinear Model Predictive Control (NMPC) scheme with a Moving Horizon Observer $(M H O)$ is presented for a Diesel engine air path. The control design based on the parameterized approach leads to a low dimensional optimization problem which makes the proposed controller real-time implementable. An attractive feature of this control strategy lies in its compatibility with more elaborated and fully nonlinear models since it uses the model as a black box predictor. 


\section{INTRODUCTION}

The production of diesel engine passenger cars has considerably increased in recent years. This success is particularly due to some important features presented in such engines when compared to standard gasoline engines: more torque at low speed, low fuel consumption, more efficiency and durability (Heywood, 1998).

On the other hand, diesel engines have important drawbacks which are the pollutant emissions. These elements consist of unburnt hydrocarbons ( $\mathrm{uHC}$ ), oxides of nitrogen, $\mathrm{NO}$ and $\mathrm{NO}_{2}$, normally referred to as $\mathrm{NO}_{x}$, carbon monoxide $\mathrm{CO}$, and particulate matter PM, mainly soot. Comparing to the gasoline engines, based on Spark-Ignition (SI) process, diesel combustion produces more $\mathrm{NO}_{x}$ and PM. On the other hand, CO emissions are negligible in diesel engines, based on Compression-Ignition (CI) process, due to lean operation and emissions of $\mathrm{uHC}$ can be handled with oxidation catalysts (Heywood, 1998; Johnson, 2001). In order to avoid PM, it is quite interesting to work with high temperatures to burn-up the soot at the boundary of the diffusion flame sheath. On the other hand, high temperatures may lead to the formation of $\mathrm{NO}_{x}$ (Guzzella and Onder, 2010). This paradox is also called $\mathrm{NO}_{x}$-PM trade-off, as shown in Figure 1 together with the maximum admissible values according to the European norms Euro III, IV and V. Therefore, the emissions of $\mathrm{NO}_{x}$ and $\mathrm{PM}$ deserve a special attention when diesel engines are considered.

For this reason, the study of diesel engine emissions has become extremely important in recent years, especially for $\mathrm{PM}$ and $\mathrm{NO}_{x}$, due to the more restrictive legislation. In order to deal with the levels of emissions, diesel engines

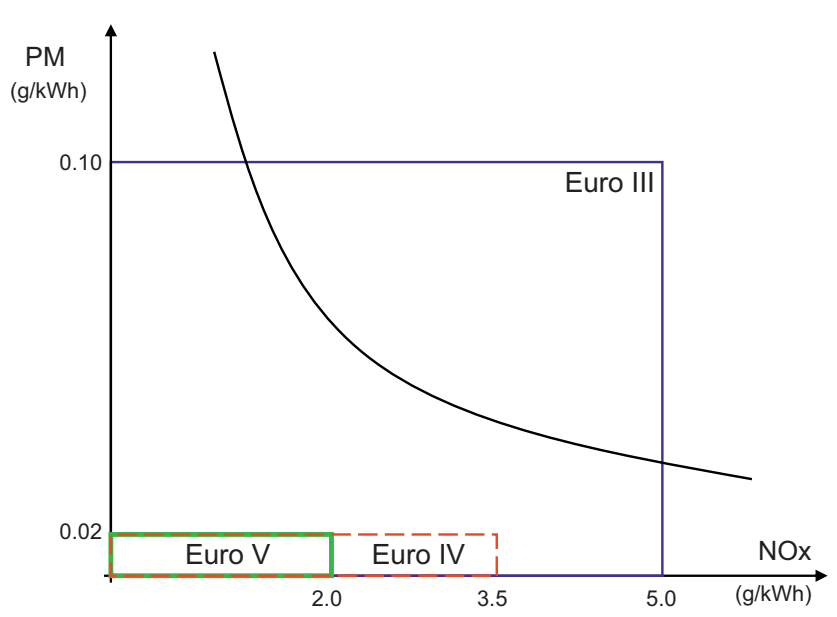

Figure 1

Schematic view of the trade-off between $\mathrm{NO}_{x}$ and PM for Diesel vehicle. are now equipped with two valves: the Exhaust Gas Recirculation (EGR) and the Variable Geometry Turbocharger (VGT). The aim is to track some dynamic setpoint trajectory on the Mass Air Flow (MAF) and the Manifold Absolute Pressure (MAP) of the engine. Therefore, a good tracking of the MAF and the MAP leads to a low level of emissions. The complete explanation and more details about the effects of EGR and VGT on the emissions control can be found in Jacobs et al. (2003) and van Nieuwstadt et al. (2000).

In order to deal with diesel engine emissions, the process to be controlled concerns the engine air path. Figure 2 shows a simplified view of the diesel engine air path. The arrows indicate the direction of the air path into the engine. The main variables used for control design involves two valves which are the manipulated variables, namely EGR and VGT, two measured disturbances, fuel injection $w f$ and the engine speed $N$ and the two already mentioned outputs, namely the MAF and the MAP. Normally, the set-points of the MAF and the MAP are provided by a linear interpolation according to two-dimensional look-up table of the operational point defined by the fuel injection and the engine speed, in order to ensure a good level of emissions.

Several control strategies have been proposed to address the above problem. From a control point of view, the diesel engine is a highly coupled multi-variable system due to the combined effects of VGT and EGR (Nieuwstadt et al., 1998). Moreover, the system is quite nonlinear (which is shown in the forthcoming sections) and constrained. Therefore, sophisticated controllers are necessary to correctly address the underlying control problem. In Jankovic and Kolmanovsky (1998) a nonlinear robust controller is presented by using standard Lyapunov-like approaches and feedback linearization. The flatness property introduced by Fliess et al. (1992) inspired some other nonlinear strategies

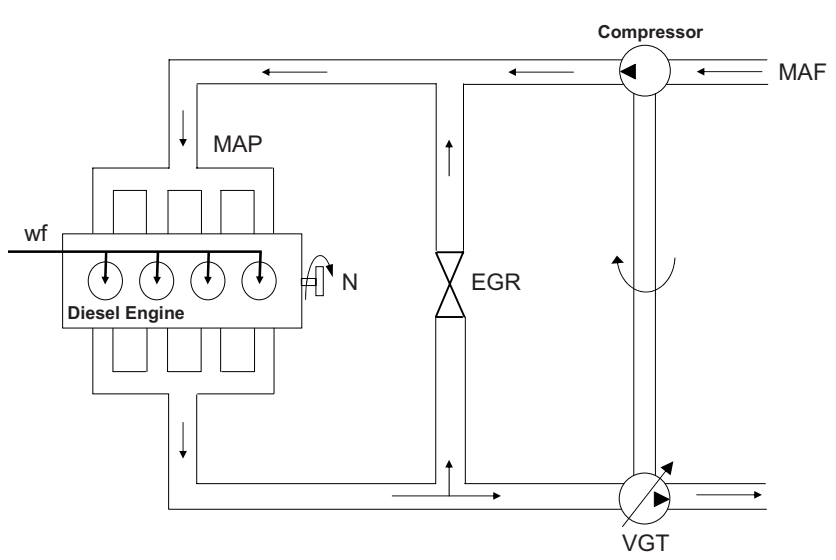

Figure 2

Simplified schematic view of the diesel engine air path and the variables used for control design. 
as in Plianos and Stobart (2007) and Chauvin et al. (2008). Robust controller is also invoked by Jung (2003) based on linearization of a Mean Values Model (MVM).

Since diesel engines are highly nonlinear and constrained systems, Nonlinear Model Predictive Control (NMPC) arises as an interesting issue for such scenario (Mayne et al., 2000). However, real time implementation is the major drawback of NMPC-like approaches, since in most of the cases, finding the optimum solution may require a huge computation effort, leading to infeasible controllers. Model uncertainties may also represent a disadvantage for NMPC if they are not considered somewhere in the problem formulation.

Some MPC approaches have already been developed for the diesel engine. In Ortner and del Re (2007), an explicit formulation is used, and some experimental results are presented. Nevertheless, since a huge look-up table is needed to address twelve local linear models, the effort to make all the off-line calculations limits the range of the control horizon $n_{c}$, which is therefore set to 1 . On the other hand, Ferreau et al. (2007) proposes a general purpose active set based controller where on line computations are performed. The approach is still based on linear models, but a higher control horizon is allowed in this case $\left(n_{c}=5\right)$.

In this paper, an output feedback strategy based on a Moving Horizon Observer (MHO) and a parameterized NMPC is proposed for a diesel engine test bench ${ }^{(1)}$. Some simulation results of the present strategy were presented in Murilo et al. (2009). The main point of this control design is the fact that the optimal solution computed by the predictive controller is completely independent of the nonlinear model structure being used, which may lead to a kind of blackbox solution for diesel engines. Moreover, the constraints on the control inputs are structurally taken into account in the formulation and the optimization routine is reduced to a simple low dimensional optimization problem enabling very short computation times to be obtained.

This paper is organized as follows. In Section 1, the system model is introduced. Then, Section 2 presents the MHO to be used in the output feedback strategy. The control design and the parameterized NMPC scheme are shown in Section 3. Section 4 shows the experimental validation on a diesel engine test bench. Finally, conclusions and future works are discussed in Section 5.

\section{SYSTEM MODEL AND CONTROL PROBLEM}

Modeling of diesel engines is still an open issue. Several approaches have been developed. In most of them (Christen et al., 2001; Jung, 2003), a mean value model is adopted to represent the system dynamics. Very often, the control design is based on linearized and Linear Parameter Varying

(1) Euro 4 passenger car diesel engine, at Johannes Kepler University Linz.
(LPV) models that are derived using a mean value model (Wei, 2006). However, since diesel engines show strong nonlinearities and active constraints on the inputs have to be correctly handled, standard multi-linear methods may be insufficient to cover the whole engine operational range and the use of fully nonlinear models may become a crucial issue. Control design methods that can cope with such models become therefore attractive. The aim of this section is to present such an alternative using two identified data models. The first one is linear up to an output injection while the second is linear up to an input injection. The identification of both methods were previously obtained using a Euro 4 passenger car diesel engine test bench available at the Johannes Kepler University (JKU) Linz and the details about system identification are more explained in Ortner et al. (2009). These two models are presented in the next section.

\subsection{Nonlinear Model Depending on the Outputs}

This model involves a state vector of dimension 14 and has been obtained using a sampling period of $10 \mathrm{~ms}$. It shows the following structure:

$$
\begin{aligned}
x^{+} & =\left[A\left(y-y_{c}\right)\right] x+B_{1}\left[u-u_{c}\right]+B_{2}\left[w-w_{c}\right] \\
y & =\left[C\left(y-y_{c}\right)\right] x+y_{c}+\varepsilon \\
\varepsilon^{+} & =\varepsilon
\end{aligned}
$$

where $x \in \mathbb{R}^{n_{x}}$ is the state $\left(n_{x}=14\right), y \in \mathbb{R}^{n_{y}}, u \in \mathbb{R}^{n_{u}}$ and $w \in \mathbb{R}^{n_{w}}$ the vector of output, control and measured disturbances respectively $\left(n_{w}=n_{y}=n_{u}=2\right)$. Finally, $\varepsilon \in \mathbb{R}^{n_{y}}$ represents the current estimation of the prediction error that enables uncertainties and model mismatches to be handled. The updating law for this variable is discussed in Section 2. The terms $u_{c}, y_{c}$ and $w_{c}$ represent central values of input, output and disturbances respectively at the operation point that has been used to identify the model.

The matrices $B_{1} \in \mathbb{R}^{n_{x} \times n_{u}}$ and $B_{2} \in \mathbb{R}^{n_{x} \times n_{w}}$ are constant while $A(\cdot) \in \mathbb{R}^{n_{x} \times n_{x}}$ and $C(\cdot) \in \mathbb{R}^{n_{y} \times n_{x}}$ depend on the output vector $y$. These matrices have been identified at a set of central values $y_{c}$. This clearly makes the present model nonlinear. However, the pair $[A(\cdot), C(\cdot)]$ is not completely observable as it will be discussed later and simulation results using this model have been presented in Murilo et al. (2009). For the experimental validation, a second model has been identified that is presented in the next section.

\subsection{Nonlinear Model Depending on the Inputs}

The second model (Ortner et al., 2009) shows the same structure than before except that the system matrices depend on the input rather than on the output. The resulting model involves 8 states and has been obtained using a sampling period of $50 \mathrm{~ms}$. More precisely:

$$
\begin{aligned}
x^{+} & =\left[A\left(u-u_{c}, w-w_{c}\right)\right] x+B_{1}\left[u-u_{c}\right]+B_{2}\left[w-w_{c}\right] \\
y & =\left[C\left(u-u_{c}, w-w_{c}\right)\right] x+y_{c}+\varepsilon \\
\varepsilon^{+} & =\varepsilon
\end{aligned}
$$


where $x \in \mathbb{R}^{n_{x}}$ is the state $\left(n_{x}=8\right)$. The matrices $B_{1}$ and $B_{2}$ are constant while $A(\cdot)$ and $C(\cdot)$ are now dependent on inputs $u$ and $w$ instead of $y$. The outputs, inputs and measurement disturbances are unchanged $\left(n_{x}=n_{y}=n_{u}=2\right)$.

One of the advantages of (2) when compared to the output dependent model (1) lies in the fact that the pair $[A(\cdot), C(\cdot)]$ is observable for each value of the control in the operational region of interest. Moreover, it covers more operational points of the engine and leads to more accurate results according to Ortner et al. (2009). For this reason, model (2) was chosen by the JKU Linz for the experimental validation and is used hereafter for control and observer design. On the other hand, the model (1) corresponds to a sampling period of $10 \mathrm{~ms}$ compared to $50 \mathrm{~ms}$ for the model given by (2). This would have been interesting in deriving a more reactive controller.

\subsection{The Control Problem}

The control problem is to design an output feedback that forces the output $y$ (namely the MAF and the MAP) to track some desired set-point $y^{d}$. Moreover, for infeasible values of $y^{d}$, the controller must stabilize the system at the closest admissible value. This has to be done while meeting the constraints on the input vector $u$ (represented by the valves EGR and VGT), namely:

$$
\begin{aligned}
u & \in\left[u_{\text {min }}, u_{\text {max }}\right] ; \quad u_{\text {min }} \in \mathbb{R}^{2} ; \quad u_{\text {max }} \in \mathbb{R}^{2} \\
\delta u & \in\left[-\delta_{\text {max }},+\delta_{\text {max }}\right] ; \quad \delta_{\text {max }} \in \mathbb{R}^{2}
\end{aligned}
$$

where $\delta u(k)=u(k)-u(k-1)$ are the increments on the inputs. Note that the inclusions in (3) and (4) are to be interpreted componentwise.

\subsection{Model Particularities}

The diesel engine model shows two properties that are worth considering in the control design: These are the non minimum phase and the open-loop stability.

\subsubsection{Non-Minimum Phase}

This property is related to the presence of high coupling in the underlying phenomenon. To illustrate this feature through a simple example, one may note that when the EGR valve is opened, more exhaust gas recirculates into the intake manifold resulting in a slight increase in the MAP. In parallel to this, less exhaust gas goes to the turbine, which slows down the turbocharger, and hence decreases the MAP. Such situations are very common in diesel engines, introducing a non-minimum phase behavior.

Basically, the non-minimum phase behavior can be viewed on the output's profile, as shown in Figure 3, namely, the outputs are forced to go to the opposite direction to the desired one during a transient phase before reaching the setpoint. As long as NMPC design is concerned, this implies

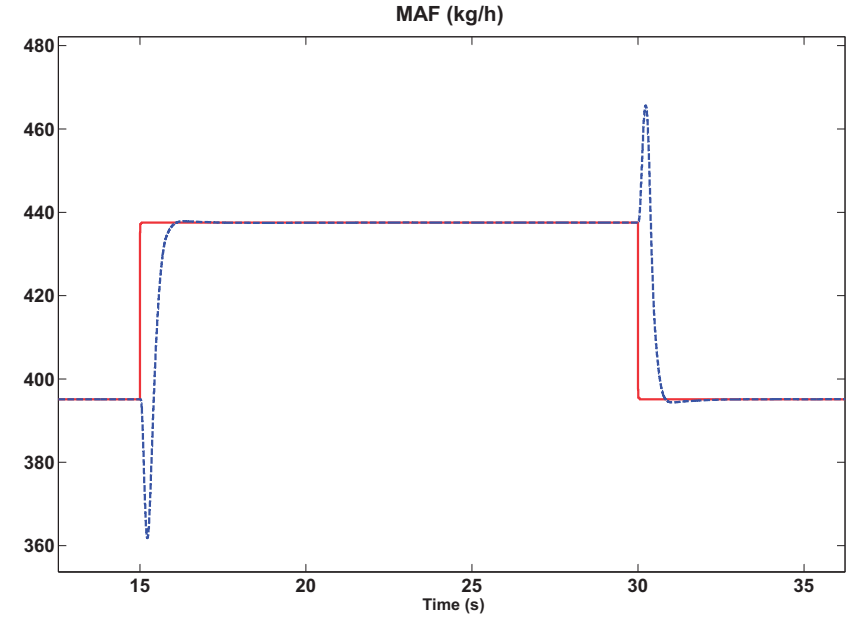

Figure 3

Simulation illustrating the non-minimum phase of the mass air flow.
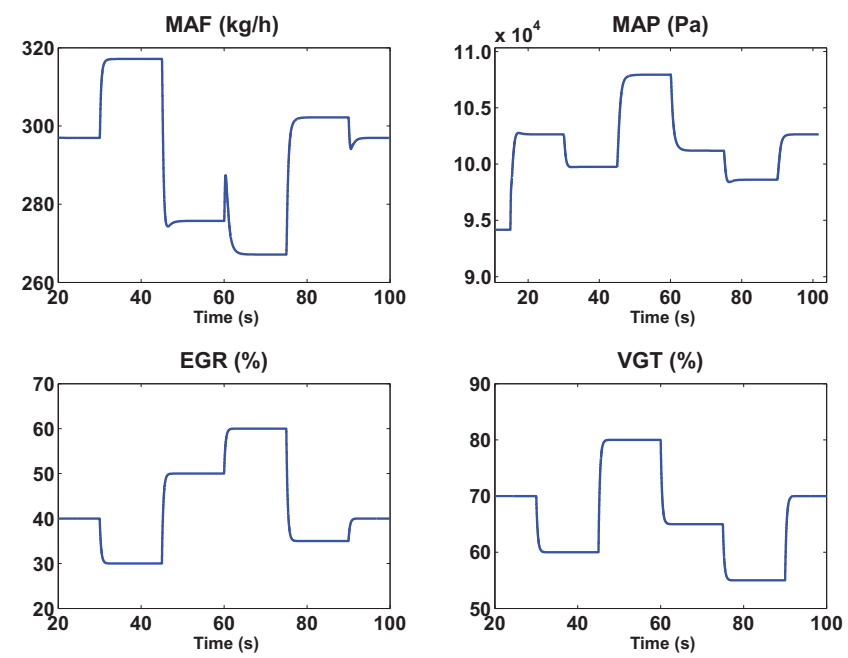

Figure 4

Open-loop behavior at $N=1800 \mathrm{RPM}$ and $w f=20 \mathrm{mg} / \mathrm{cyl}$ under a step sequence of EGR and VGT.

that the prediction horizon must be sufficiently high to go beyond this transient phase. On the other hand, increasing the prediction horizon too much leads to computation time that can go beyond what can be performed given the available computation time. A trade-off is required here to combine these two contradictory effects.

\subsubsection{Open-Loop Behavior}

A second important point to emphasize concerns the openloop behavior of the diesel engine model. As a matter of fact, the system is open-loop stable. Figure 4 illustrates the outputs when a sequence of step changes is used as inputs. 
This means that for a given value of the EGR, the VGT, the speed and the fuel injection, the outputs reach a given set of steady values.

The open-loop stability can be used in the NMPC design by computing first the steady state to be reached and then use the remaining degrees of freedom to improve the quality of the transient. This is more detailed in Section 3. But let us first introduce the observer used to recover the entire state of the system.

\section{OBSERVER DESIGN}

\subsection{The Importance of the Observer}

The NMPC design needs the state of the system to be reconstructed. As a matter of fact, the first nonlinear model (1) depending on the outputs is not observable. Nevertheless, a moving horizon algorithm can be used to reconstruct the observable part of the system. Note that this may not be sufficient in general. Here it could be enough since the problem is to track the desired set-point of the measured output and thanks to the open-loop stability property of the system model. As as result, for the model (1), a MHO was developed, as shown in Murilo et al. (2009), in order to deal with this lack of observability where other classical observers like Extended Kalman Filter (EKF) are inappropriate.

However, it can be shown that the second nonlinear model (2) which is used here in the experimental validation is observable for any input vector belonging to the set of operational values of interest. As a result, the same structure of the MHO, initially developed for the first model (1) and presented in Murilo et al. (2009) is also adopted in this paper to reconstruct the state of (2). This is detailed in the next section.

\subsection{Moving Horizon Observer Design}

Moving horizon observers share the same advantages and drawbacks of NMPC (Alamir, 1999; Alamir and CalvilloCorona, 2002; Michalska and Mayne, 1995). Indeed, these observers can deal with nonlinearities and constraints in the system at the price of on-line optimization that may be incompatible with the available computation time.

The main idea of MHO's is to estimate the current state using a moving and fixed-size window containing $N_{o}$ past measurements. The oldest measurements are discarded while the newest one is taken into account. Then, the collected data are used to define a cost function, which is minimized with the state at the beginning of the observation window as a decision variable. As mentioned previously, the observer was developed using the model (2) which is used hereafter in the experimental validation.

Let us first consider a fixed-size window containing $N_{o}$ past measurements. At the present instant $k$, the value of the current state $x(k)$ can be derived from the past state $x^{(-)}=$ $x\left(k-N_{o}+1\right)$ at the beginning of the observation horizon and the vectors of past inputs $\bar{U}_{k}$ according to:

$$
\begin{aligned}
x(k) & =\left[\Phi\left(\bar{U}_{k}\right)\right] \cdot x^{(-)}+\left[\Psi\left(\bar{U}_{k}\right)\right] \cdot \bar{U}_{k} \\
\bar{Y}_{k} & =\left[\Omega\left(\bar{U}_{k}\right)\right] \cdot x^{(-)}+\left[\Gamma\left(\bar{U}_{k}\right)\right] \cdot \bar{U}_{k}+\bar{E}_{k}
\end{aligned}
$$

where $\bar{Y}_{k} \in \mathbb{R}^{n_{y} \cdot N_{o}}$ is the vector of past measurements, $\bar{U}_{k} \in \mathbb{R}^{\left(n_{u}+n_{w}\right) \cdot N_{o}}$ is the vector of control inputs and measurement disturbances while the matrices $\Phi\left(\bar{U}_{k}\right), \Omega\left(\bar{U}_{k}\right), \Gamma\left(\bar{U}_{k}\right)$ and $\Psi\left(\bar{U}_{k}\right)$ are obtained after straightforward computations in terms of the system matrices $A(\cdot), C(\cdot), B=\left[\begin{array}{ll}B_{1} & B_{2}\end{array}\right]$. The vector $\bar{E}_{k}$ corresponds to the past prediction error values represented by $\varepsilon$. In order to update the estimation of the prediction error, the value of $\varepsilon$ is updated at each sampling instant $k$ according to:

$$
\varepsilon(k)=\varepsilon(k-1)+k_{i} \cdot\left(y^{p}(k)-y^{m}(k)\right)
$$

where $y^{p}(k)$ and $y^{m}(k)$ are, respectively, the predicted value under the previous estimated state at instant $k-1$ and the measured output at instant $k, \varepsilon(k-1)$ the previous value of the prediction error and $k_{i} \in \mathbb{R}^{n_{y}}$ the integrator gain. Therefore, the past estimation $\hat{x}(k-1)$ can be related to $\hat{x}(k)$ in (5) as follows:

$$
\begin{aligned}
& A(u(k-1), w(k-1)) \hat{x}(k-1)+B[u(k-1) w(k-1)]^{T}= \\
& {\left[\Phi\left(\bar{U}_{k}\right)\right] \cdot x^{(-)}+\left[\Psi\left(\bar{U}_{k}\right)\right] \cdot \bar{U}_{k}} \\
& \bar{Y}_{k}=\left[\Omega\left(\bar{U}_{k}\right)\right] \cdot x^{(-)}+\left[\Gamma\left(\bar{U}_{k}\right)\right] \cdot \bar{U}_{k}+\bar{E}_{k}
\end{aligned}
$$

Note that these two equations represent the requirements on the past value $x^{(-)}$to be estimated. The first requirement is related to the state equation while the second is related to the measured output. Therefore, a trade-off similar to the classical one involved in the Kalman-filter through the covariance related weighting matrices can be obtained by means of the following cost function:

$$
\hat{x}^{(-)}=\arg \min _{\xi \in \mathbb{R}^{n}}\left\|G_{1} \cdot \xi-S_{1}\right\|_{Q_{1}}^{2}+\left\|G_{2} \cdot \xi-S_{2}\right\|_{Q_{2}}^{2}
$$

where $Q_{1}$ and $Q_{2}$ are the weighting matrices that can be taken equal to the inverse of the noise covariance matrices exactly as in the EKF framework, and the other matrices are defined so that the cost function expresses the above two requirement (8) and (9), namely:

$$
\begin{aligned}
G_{1}= & \Omega\left(\bar{U}_{k}\right) \\
G_{2}= & \Phi\left(\bar{U}_{k}\right) \\
S_{1}= & Y_{k}-\Gamma\left(\bar{U}_{k}\right) \cdot \bar{U}_{k}-\bar{E}_{k} \\
S_{2}= & A(u(k-1), w(k-1)) \hat{x}(k-1)+ \\
& B[u(k-1) w(k-1)]^{T}-\Psi\left(\bar{U}_{k}\right) \cdot \bar{U}_{k}
\end{aligned}
$$



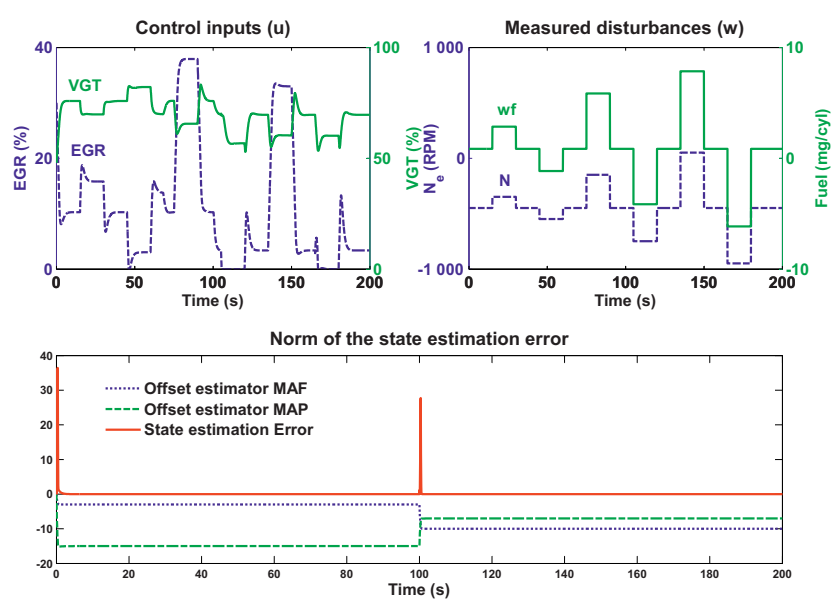

Figure 5

Simulation results related to the state estimation error and the offset estimation. The disturbance $\varepsilon$ inserted at $t=100 \mathrm{~s}$ is quickly determined by the updating mechanism (7).

With the above notations, an updating law $\hat{x}(k)$ for the state estimation can be defined as follows:

$$
\begin{array}{r}
\hat{x}(k)=\Phi\left(\bar{U}_{k}\right) \cdot\left[\left(G_{1}^{T} \cdot Q_{1} \cdot G_{1}+G_{2}^{T} \cdot Q_{2} \cdot G_{2}^{T}\right)^{\dagger}\right. \\
\left.\left(S_{1}^{T} \cdot Q_{1} \cdot G_{1}+S_{2}^{T} \cdot Q_{2} \cdot G_{2}\right)^{T}\right] \hat{x}(k-1)+\Psi\left(\bar{U}_{k}\right) \cdot \bar{U}_{k}
\end{array}
$$

leading to the following implicit form for the observer:

$$
\hat{x}(k)=\operatorname{Obs}\left(\hat{x}(k-1), \bar{Y}_{k}, \bar{U}_{k}\right)
$$

The simulation results of Figure 5 show the evolution of the norm of the state estimation error $\|x-\hat{x}\|$ of the moving horizon observer and the disturbance rejection of the offset $\varepsilon$ given by (7). The weighting matrices $Q_{1}$ and $Q_{2}$ were set to the identities and the integrator gain $k_{i}=(3.5,3.5)$. Note that despite the presence of a bias on the outputs, the asymptotic convergence of the estimation error is provided by the observer thanks to the updating mechanism (7).

\section{CONTROL DESIGN}

In this section, a parameterized NMPC scheme is proposed for the input dependent model (2). This scheme involves a state observer design presented in the previous section as well as the computation of the steady state that is compatible with the desired output. This is the aim of the next section.

\subsection{Steady State Computation}

The construction of the control parametrization involves the computation of the steady state/control pair that is compatible with the desired output value. This comes from the

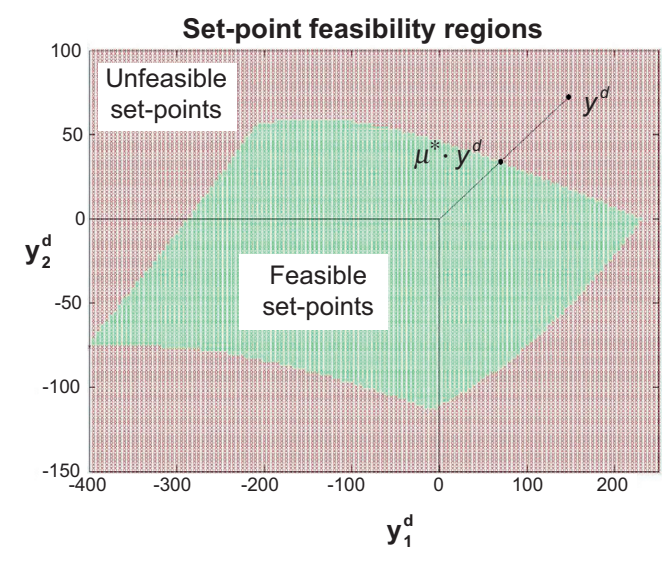

Figure 6

Achievable set-points $y^{d}$ at $w=(0,0)$ for the model (1).

fact that the diesel engine system is open loop stable, as shown previously. More precisely, given the measured disturbance vector $w$ and the desired value $y^{d}$, if the model (2) is considered, the steady control is computed by solving the following two-dimensional optimization problem:

$$
\begin{aligned}
& u^{*}\left(w, y^{d}\right)=\arg \min _{u_{d} \in\left[u_{\min }, u_{\max }\right]}\left\|y_{c}\left(u_{d}, w\right)-y^{d}\right\|^{2} \\
& y_{c}\left(u_{d}, w\right)=C\left(u_{d}, w\right)\left[\mathbb{I}_{n}-A\left(u_{d}, w\right)\right]^{-1} .\left[B \cdot u_{d}+G . w\right]
\end{aligned}
$$

Moreover, having the steady control $u^{*}$, the steady state $x^{*}$ easily follows according to:

$$
x^{*}\left(u^{*}, w\right)=\left[\mathbb{I}_{n}-A\left(u^{*}, w\right)\right]^{-1} \cdot\left[B \cdot u^{*}+G \cdot w\right]
$$

As a matter of fact, the present formulation represents a generic way to find the stationary control (11). By keeping this simple optimization problem, one enforces the generic definition of the control structure. In the next section, it is shown how this steady state pair is incorporated in the NMPC control design in order to derive a real-time compatible output feedback scheme. However, before going further into the NMPC formulation, it is important to emphasize some notions about the feasibility of set-points.

As mentioned in the introduction, the set-points $y^{d}$ are generated by another control loop that manages the emission level. Considering for instance the model (1) and assuming that the set-point $y^{d}$ is given as well as the measured disturbance vector $w$. In Murilo et al. (2009), it has been shown that one can define a Linear Programming (LP) problem according to (1), in order to compute the closest feasible setpoint corresponding to the steady state $x^{*}$. The parameter $\mu^{*}$ is obtained by solving this LP and gives a crucial information on the feasibility of the desired set-points. This can be clearly seen in Figure 6, where a schematic view of the set of achievable values $y^{d}$ under $w=0$ is shown. 
The green region of Figure 6 shows the achievable zones, those for which the solution of the $L P$ gives $\mu^{*}=1$. No controller can achieve a pair that is outside this region. If a set-point falls in the red region it must be replaced by the closest one $y^{*}=\mu^{*} \cdot y^{d}$ corresponding to $\left(0 \leq \mu^{*}<1\right)$. The formulation of the related LP and the complete description of its parameters are presented in Murilo et al. (2009).

\subsection{Recall on the Parameterized NMPC}

It is well-known that one of the main challenges concerning NMPC approaches is the real-time implementation. This is much more evident when fast dynamical systems are considered since optimization process must be solved within the sampling time. Many approaches have been developed in recent years to meet the real-time implementation requirement. Although different kinds of classifications can be adopted. One possible and legitimate classification splits the existing solution in two categories: Piece-Wise Affine-like (PWA) solutions (Bemporad et al., 2002; Ferreau et al., 2006) and fully nonlinear solution (Alamir, 2006; Diehl et al., 2005; Ohtsuka, 2004; Zavala et al., 2006).

The approach proposed in the present papers falls in this last category. More precisely, the parameterized approach is adopted here as it enables a low dimensional on-line optimization problem to be derived that can be solved using simple and therefore potentially certified solution. This last feature is of great importance when talking about solutions that have to be adopted in industrial large production units context. Indeed, in such context, implementing quite involved algorithms that would be needed to handle high dimensional decision variable may be incompatible with car company certification requirements.

The control parametrization approach comes down to choose the candidate piecewise open-loop control profiles within a class of control profiles that are defined by a low dimensional vector of parameters $p \in \mathbb{P}$. More precisely, a map $\mathcal{U}_{p w c}: \mathbb{P} \times \mathbb{R}^{n_{x}} \rightarrow \mathbb{U}^{N_{p}}$ has to be defined where $N_{p}$ is the prediction horizon, such that the piecewise constant control sequence corresponding to the parameter $p$ is given by:

$$
\mathbf{u}=\mathcal{U}_{p w c}(p, \hat{x}(k))
$$

where $p$ is the parameter to optimize at each decision instant $k$, and $\hat{x}(k)$ the estimated state at present instant.

Now, the optimal parameter $\hat{p}$ is computed by solving the following optimal control problem in the decision variable $p$ for a given estimated state $\hat{x}(k)$ :

$$
\hat{p}=\arg \min _{p \in \mathbb{P}}[J(p, \hat{x}(k))] \quad \text { under } \quad C(p, \hat{x}(k)) \leq 0
$$

where $C(p, \hat{x}(k))$ are the constraints to be respected and $\hat{p}$ the optimal parameter vector. Once the optimal parameter $\hat{p}$ is obtained, the optimal control sequence $\tilde{\mathbf{u}}$ follows according to:

$$
\tilde{\mathbf{u}}=\mathcal{U}_{p w c}(\hat{p}, \hat{x}(k))=\left(u^{(1)}(\hat{p}, \hat{x}(k)) \ldots u^{\left(N_{p}\right)}(\hat{p}, \hat{x}(k))\right)
$$

from which only the first element of $u^{(1)}(\hat{p}, \hat{x}(k))$ is applied during the sampling period $[k+1, k+2]$, namely:

$$
u^{o p t}(k+1)=u^{(1)}(\hat{p}, \hat{x}(k))
$$

During the next sampling period $[k+1, k+2]$ (while the control $u^{o p t}(k+1)$ is applied), the same steps described above are executed again, and the next optimal control namely $u^{o p t}(k+2)$ is provided, which leads to an implicit output feedback strategy. In the following section, the parametrization used to solve the diesel engine control problem as described above is presented.

\subsection{Parametric NMPC Formulation for Diesel Engines}

The parametrization of the control sequence involves the stationary control (11) together with a simple temporal parametrization of the future control sequence. In order to do this, at the current instant $k$, the controller needs the estimated state $\hat{x}(k)(10)$, the actual value of the control input $u(k-1)$, already scheduled according to the computation performed over the sampling period $[k-1, k]$ and the values of the measured disturbances $w(k)$.

While in standard formulation, all the components of the control sequence $\mathbf{u}$ are taken as degrees of freedom, here an exponential parametrization is used (Alamir, 2006). More precisely, the temporal structure of the control sequence can be written as follows:

$$
\begin{array}{r}
\mathbf{u}\left(i \tau_{s}+t\right)=S a t_{u_{\min }}^{u_{\max }}\left(u^{*}+\alpha_{1} \cdot e^{-\lambda \cdot i \cdot \tau_{s}}+\alpha_{2} \cdot e^{-q \cdot \lambda \cdot i \cdot \tau_{s}}\right) \\
\text { for } t \in\left[(k-1) \tau_{s}, k \tau_{s}[\right.
\end{array}
$$

where $i \in\left\{1, \ldots, N_{p}\right\}, \tau_{s}$ is the sampling time, $\lambda>0, q \in \mathbb{N}$ are tuning parameters, $\alpha_{1}, \alpha_{2} \in \mathbb{R}^{n_{u}}$ are the coefficients to be determined as explained below and $S$ at a saturation map for the control input $S$ at $: \mathbb{R}^{n_{u}} \rightarrow \mathbb{R}^{n_{u}}$ defined as:

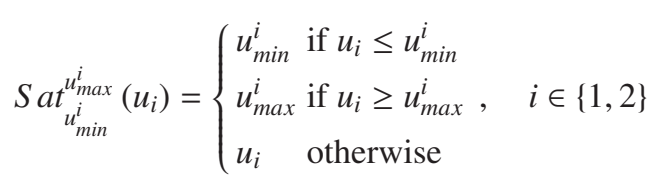

Note that Equation (13) leads to the following set of equations:

$$
\begin{aligned}
& u^{*}+\alpha_{1}+\alpha_{2}=u(k-1) \\
& \alpha_{1} \cdot\left(e^{-\lambda \cdot \tau_{s}}-1\right)+\alpha_{2} \cdot\left(e^{-q \cdot \lambda \cdot \tau_{s}}-1\right)=p \cdot \delta_{\max }
\end{aligned}
$$

where the first equation guarantees continuity of the control profiles when $i=0$ in (13) while the (16) simply states that the difference between two successive applied control does not exceed a fraction $p \in[-1,1]^{2}$ of some maximal allowable values $\delta_{\max } \in \mathbb{R}^{n_{u}}$ involved in the constraint (4). 
The terms $\alpha_{1}$ and $\alpha_{2}$ become now dependent on the parameter vector $p$ and can be determined by:

$$
\begin{gathered}
\left(\begin{array}{c}
\alpha_{1}^{u_{1}}(p) \\
\alpha_{2}^{u_{1}}(p) \\
\alpha_{1}^{u_{2}}(p) \\
\alpha_{2}^{u_{2}}(p)
\end{array}\right)=\left(\begin{array}{cccc}
1 & 1 & 0 & 0 \\
e^{-\lambda . \tau_{s}}-1 & e^{-q . \lambda . \tau_{s}}-1 & 0 & 0 \\
0 & 0 & 1 & 1 \\
0 & 0 & e^{-\lambda \cdot \tau_{s}}-1 e^{-q \cdot \lambda \cdot \tau_{s}}-1
\end{array}\right)^{-1} \\
\left(\begin{array}{c}
u_{1}(k-1)-u_{1}^{*} \\
p_{1} \delta_{\max } 1 \\
u_{2}(k-1)-u_{2}^{*} \\
p_{2} \delta_{\max }^{2}
\end{array}\right)
\end{gathered}
$$

where the notation $\alpha_{1}=\left[\alpha_{1}^{u_{1}} ; \alpha_{1}^{u_{2}}\right]$ and $\alpha_{2}=\left[\alpha_{2}^{u_{1}} ; \alpha_{2}^{u_{2}}\right]$ is used. Injecting (17) in the expression of the control sequence (13) leads to:

$$
\mathbf{u}\left(i \tau_{s}+t, p\right)=S a t_{u_{\min }}^{u_{\max }}\left(u^{*}+\alpha_{1}(p) \cdot e^{-\lambda \cdot i \cdot \tau_{s}}+\alpha_{2}(p) \cdot e^{-q \cdot \lambda \cdot i \cdot \tau_{s}}\right)
$$

Based on the above notation, given the closest feasible setpoint $y^{*}$, the estimated state $\hat{x}$ and the current measurement $w$ of the disturbance vector, the best set of parameters $\hat{p}$ to be used in the definition of optimal control sequence is provided by solving the following optimization problem:

$$
\begin{array}{r}
\hat{p}=\arg \min _{p \in \mathbb{P}}\left[\rho_{x} \cdot\left\|X\left(N_{p}, \hat{x}(k), p\right)-x^{*}\left(y^{*}, w\right)\right\|+\right. \\
\left.\sum_{i=0}^{N_{p}-1}\left\|Y(i, \hat{x}(k), p)-Y_{f}^{*}\left(i, y^{*}\right)\right\|_{Q_{y}}^{2}\right]
\end{array}
$$

where $Y(i, \hat{x}(k), p)$ and $X\left(N_{p}, \hat{x}(k), p\right)$ are respectively, the output and the final state prediction based on the system model (2) under the open loop control profile defined by $p$ over $\left[k+1, k+N_{p}\right]$ and starting from the estimated state $\hat{x}(k)$. The state $x^{*}\left(y^{*}, w\right)$ is the closest stationary state obtained by (12), $\rho_{x}>0$ is some weighting coefficient used to enforce the constraint on the final state and hence stability of the closed-loop system. Finally $Y^{f}\left(i, y^{*}\right)$ is the filtered version of the set-point trajectory enabling to decouple the response time from overshoots:

$$
Y^{f}\left(i, y^{*}\right)=y^{*}+e^{-3 \tau_{s} \cdot i / t_{r}} \cdot\left[y(k)-y^{*}\right]
$$

where $t_{r}$ is the desired response time of the closed-loop system. The weighting matrix $Q_{y} \in \mathbb{R}^{n_{y} \times n_{y}}$ is used to differently weight the outputs $y_{1}$ and $y_{2}$, and has the following structure

$$
Q_{y}=\left(\begin{array}{cc}
\frac{\rho_{1}}{\bar{y}_{1}^{2}} & 0 \\
0 & \frac{\rho_{2}}{\bar{y}_{2}^{2}}
\end{array}\right)_{n_{y} \times n_{y}}
$$

where $\rho_{1}$ and $\rho_{2}$ are the weighting terms of $y_{1}$ and $y_{2}$ respectively while $\bar{y}_{1}$ and $\bar{y}_{2}$ are normalization terms related to $y_{1}$ and $y_{2}$ respectively. Figure 7 illustrates the whole output feedback strategy, with the MHO representation and the parameterized NMPC.

The next step consists in defining a suitable optimization process in order to solve the optimization problem (18) and to obtain $\hat{p}$.

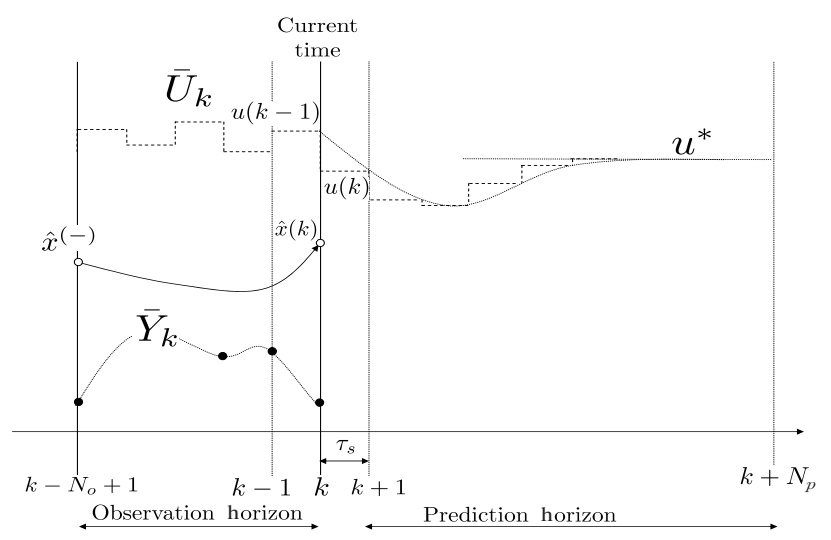

Figure 7

Schematic view showing the output feedback strategy based on the moving horizon observer and the predictive control. The present instant is $k . \bar{Y}_{k}$ is collected during the past time interval $\left[\left(k-N_{o}+1\right) \tau_{s}, k \tau_{s}\right]$ and the past values of $u$ and $w$ are stored in $\bar{U}_{k}$. Then, the state $\hat{x}(k)$ can be estimated by using (10), which is used to define the parameterized NMPC scheme.

\subsection{Optimization Process}

As soon as systems with fast dynamics are concerned, the computation time becomes a crucial issue since the time needed to reach an optimal solution may be greater that the nominal control updating period (Alamir, 2006). More precisely, assume that only a finite number $q_{\max } \in \mathbb{N}$ of iterations of some optimization process $\mathcal{S}$ can be performed during the sampling period $[k-1, k]$. The optimal parameter vector is no more available and $p$ becomes a dynamic variable that is no more defined as a function of the state. This leads to the following extended representation:

$$
\begin{aligned}
& \hat{x}^{+}(k)=X\left(\tau_{s}, \hat{x}(k), p(k)\right) \\
& p^{+}(k)=\mathcal{S}^{q_{\max }}(p(k), \hat{x}(k))
\end{aligned}
$$

where $\mathcal{S}^{q_{\max }}$ denotes $q_{\max }$ successive iterations of $\mathcal{S}$ starting from the initial guess $p(k)$, and $X\left(\tau_{s}, \hat{x}(k), p(k)\right)$ the state at instant $k+1$ under the control defined by $p(k)$.

Note that the stability of the extended system (19)-(20) depends on both the quality of the model and the efficiency of the optimizer at the current state. More detailed description of this stability issue can be found in Alamir (2009).

Figures 8 and 9 show the evolution of $p$ as well as the system outputs. Three particular instants $k_{i}, i\{1,2,3\}$ have been chosen to illustrate the shape of the performance index $J\left(\cdot, \hat{x}\left(k_{i}\right)\right)$ as a function of the two decision variables $p_{1}$ and $p_{2}$.

Regardless the solver choice, the complexity of the optimization task heavily depends on the problem formulation, in particular, the control parametrization. Figure 10 clearly shows how the low dimensional control parametrization 


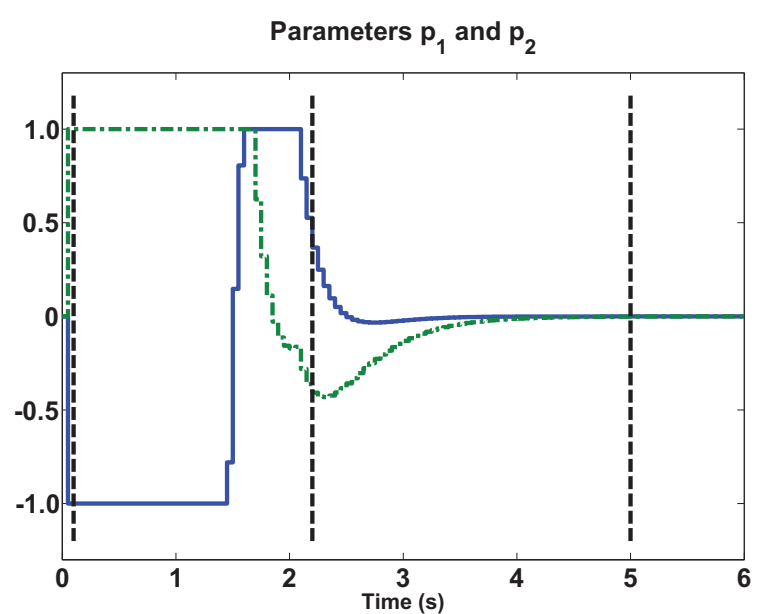

Figure 8

Simulation results showing the evolution of $p_{1}$ and $p_{2}$, with $N_{p}=30, \lambda=1, q=5, w=(448,0.8)^{T}$ and $y^{d}=(60,-25)^{T}$ (Fig. 9). The initial state of the observer has been set to 0 . Three particular instants $(t=0.1 \mathrm{~s}, t=2.2 \mathrm{~s}$ and $t=5 \mathrm{~s})$ have been used to illustrate the instantaneous shape of cost function (Fig. 10).

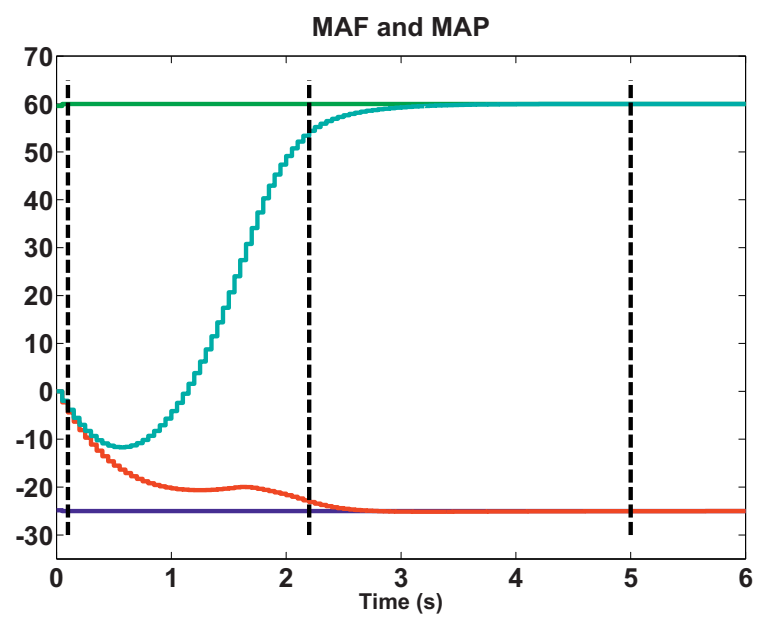

Figure 9

Simulation results showing the measured values of the MAF and the MAP (deviations from the central values) and desired outputs $y^{d}$. Vertical lines define the three instants used to illustrate the shape of the cost function (Fig. 10).

adopted above leads to a well posed optimization problem (the cost function seems to be convex). This explains why the upper bound $\left(q_{\max }=30\right)$ on the number of function evaluations has never been reached during all the forthcoming validation results.

As for the optimizer being used, some classical gradientfree algorithms such as the Powell's method and the simplex
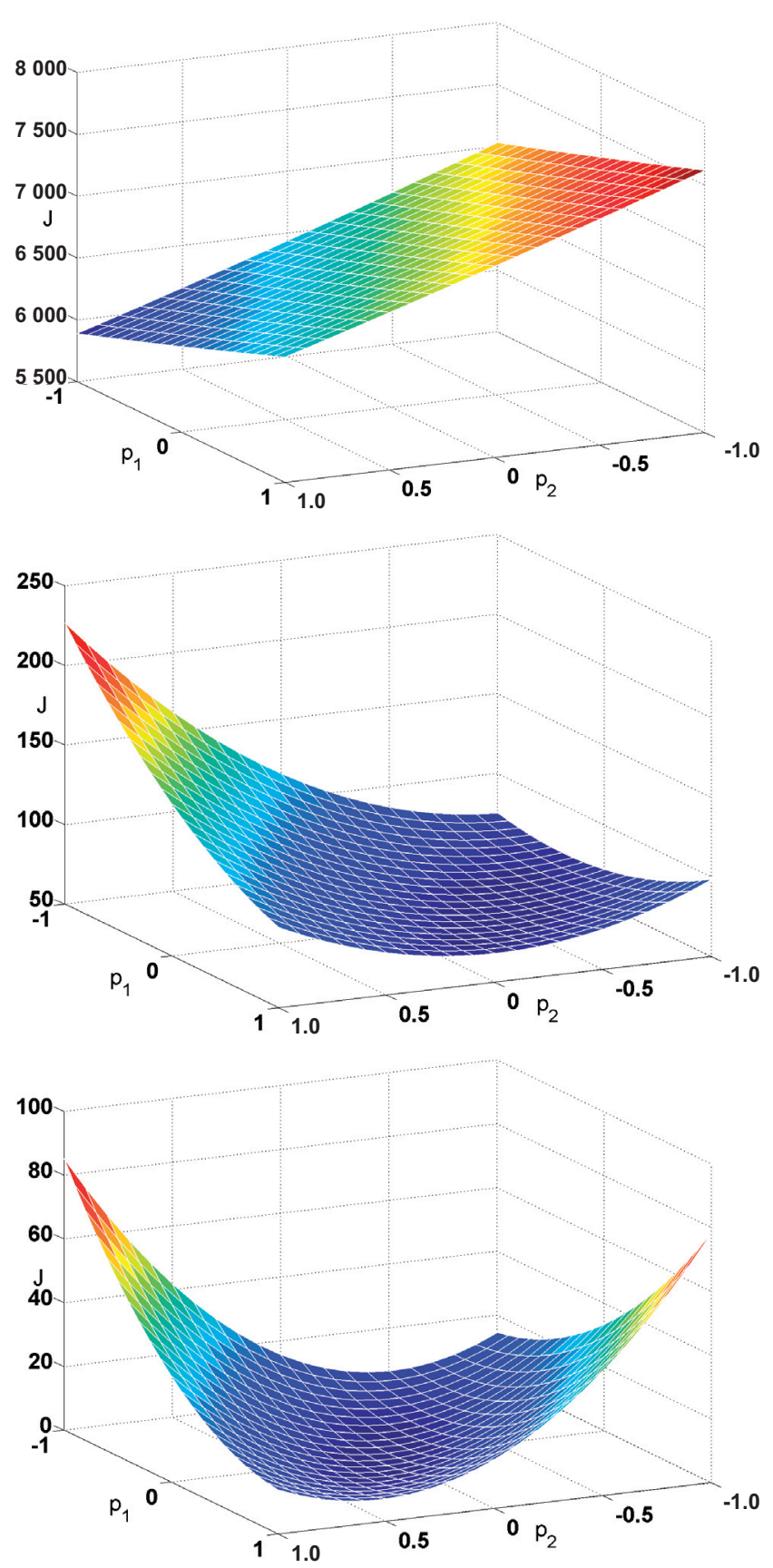

Figure 10

The shape of the cost function $J\left(\cdot, \hat{x}\left(k_{i}\right)\right)$ for the three particular instants depicted in Figures 8 and $9\left[t_{1}=0.1 \mathrm{~s}\right.$ (top), $t_{2}=$ $2.2 \mathrm{~s}$ (middle), $t_{3}=5.0 \mathrm{~s}$ (bottom)]. Note the trajectory of the optimum value $\hat{p}$ and the decrease of the cost function from top to bottom showing the efficiency of proposed algorithm. This efficiency relies on the convex shape and the low dimensional underlying optimization problem.

were tested, and the results were quite positive and very similar. For the sake of completeness, let us briefly introduce 
a particular SQP routine which was developed for simulation and also used for experimental validation. Basically, the algorithm performs successively scalar SQP's on each component, each followed by an adaptation of a trust region. After each complete cycle of scalar SQP's (over the two components), the results are used to construct an approximation of the gradient along which potentially successful steps are attempted. Again, a trust region along the gradient is updated accordingly. This yields an optimization algorithm that uses the model as a black-box simulator and is therefore easily re-usable if a more sophisticated and faithful process model is made available.

\section{EXPERIMENTAL RESULTS}

The experimental validation of the present parameterized NMPC was performed on the real world diesel engine at JKU Linz. The test bench consists in a diesel engine fulfilling the EU4 emission standard, controlled by the Engine Control Unit (ECU) and an AVL dynamometer to simulate the load on the engine shaft. The real time environment was managed by d-Space Autobox running at $480 \mathrm{MHz}$, linked to Matlab software, and the programs (controller and observer) were developed in $\mathrm{C}$ language. The sampling time was set to $50 \mathrm{~ms}$.

As mentioned earlier, the set-points are generated by look-up tables of the measured disturbances $w$, the engine speed and fuel injection. Two types of experiments are presented hereafter. First, a manual setting of $w$ profile was used, by imposing a step sequence trajectory for both variables. In the second part, the trajectories were generated by the non-urban part of the New European Driving Cycle (NEDC). This cycle is defined to represent the typical use of a car in Europe, and the resulting performance is used, among other indicators, to assess the emission levels of car engines.

Figure 11 illustrates these scenarios. In both cases, the NMPC closed-loop performances are compared to the performance of the ECU. Table 1 shows the parameters used in the experiments. Since the model (2) was identified around a central value, the MAF and the MAP are represented by their difference with respect to this central value.

TABLE 1

Parameters used in experiments

\begin{tabular}{c|c|c|c}
\hline Parameter & Value & Parameter & Value \\
\hline$\tau_{s}$ & $0.05 \mathrm{~s}$ & $\delta_{\max }$ & $(1,1)^{T} \% / \tau_{s}$ \\
$N_{p}$ & 30 & $N_{o}$ & 10 \\
$\lambda$ & 1 & $q_{\max }$ & 30 \\
$q$ & 5 & $\rho_{x}$ & 0.001 \\
$\rho_{1}$ & 1 & $\rho_{2}$ & 1 \\
$\bar{y}_{1}$ & 1 & $\bar{y}_{2}$ & 1 \\
$t_{r}$ & $3 \cdot \tau_{s} / q \cdot \lambda$ & $k_{i}$ & $(0.6,0.01)^{T}$ \\
\hline
\end{tabular}
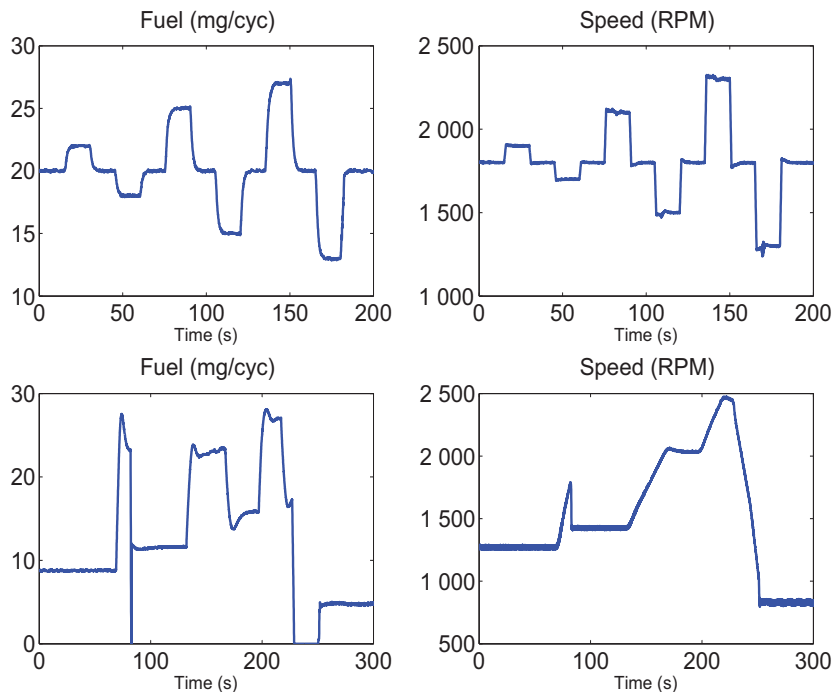

Figure 11

The measured disturbances (fuel injection and speed engine) used for the set-point generation. On the top the step sequence and on the bottom the non-urban part of the NEDC.

\subsection{Successive Steps Sequence}

Figure 12 shows the ECU's response to a sequence of step changes. The ECU's control design is based on a Single Input Single Output (SISO) controller, with feed-back control for MAF and feed-forward for MAP. As shown in this figure, the MAF is highly weighted by ECU while the MAP's tracking is viewed as a secondary task.
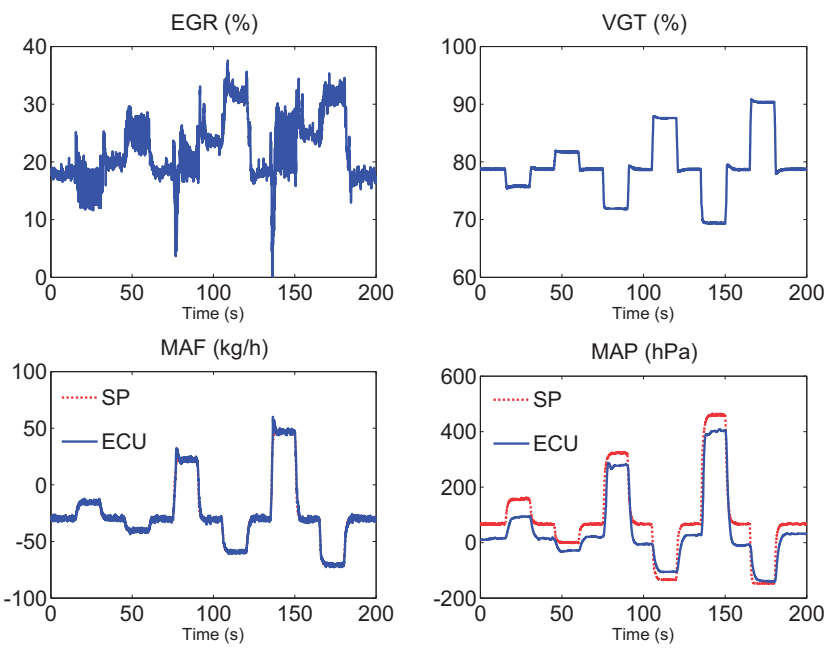

Figure 12

ECU's response to a sequence of step changes. Note that the VGT is not directly controlled and only the MAF is correctly tracked. The steady-state error for MAP can reach $90 \%$ of the desired set-point. 

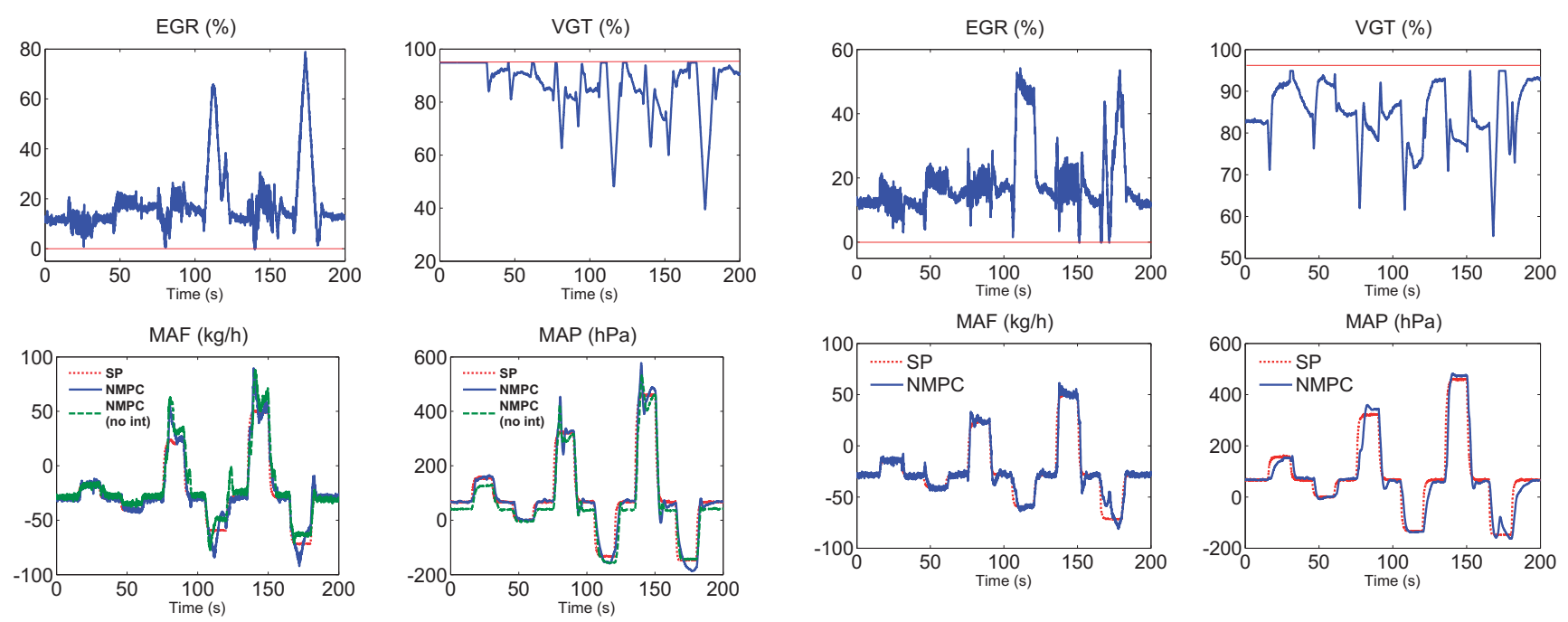

Figure 13

Experimental results using a parameterized NMPC scheme, with the standard parameters of Table 1. The MAF and the MAP are simultaneously tracked. Results are shown with and without the use of the offset estimation. It comes clearly that the integrator term eliminates the offset error in the stationary state. The overshoot can reach $70 \%$ for MAF but with a response time of 2 seconds approximately. This figure is to be compared to Figure 14 where a weighting on the terminal state has been added.

The first experimental validation of the parameterized NMPC approach is shown in Figure 13. Note that the controller is able to track both variables, which are equally weighted. The constraints on the inputs are respected, and some overshoots may come from the fact that measured disturbances are not filtered, especially the engine speed. Note the non-minimum phase behavior which forces the inputs to take one direction before coming back to the right one. Offset errors are eliminated by means of the integrator term introduced in Equation (7). Moreover, the computation time needed to perform the optimization routine never reaches the sampling rate of $50 \mathrm{~ms}$ during the runtime execution, which means that the proposed output feedback strategy is real-time implementable.

In order to deal with overshoots and oscillations, the terminal state was strongly weighted, as shown in Figure 14. On the other hand, the system's response time becomes a bit slower. This is the classical trade-off between these requirements. Some other scenarios were tested by changing the parameters of Table 1 without significant improvement on the results.

\subsection{NEDC Sequence}

The second part of the experimental validation consists in testing the present controller using the non-urban part of the
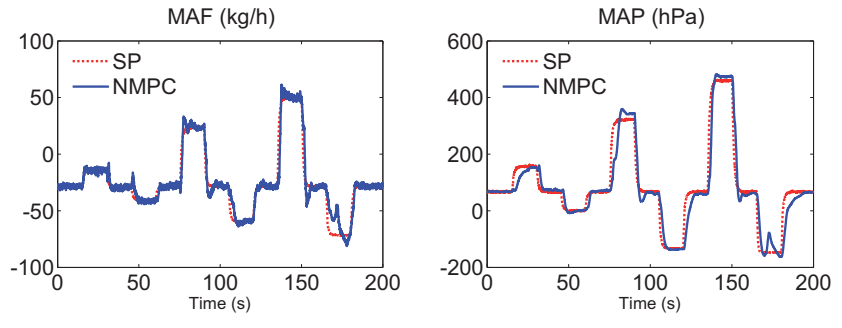

Figure 14

Experimental results using a parameterized NMPC scheme, with $\rho_{x}=1000$. Comparing to the previous scenario, overshoots are considerably reduced for both outputs (maximum of $5 \%$ for MAP) by weighting the terminal state at the price of the slower response time (interval from 5 to 15 seconds for MAP depending on the operational point of the engine). Moreover, the steady-state error is correctly handled.
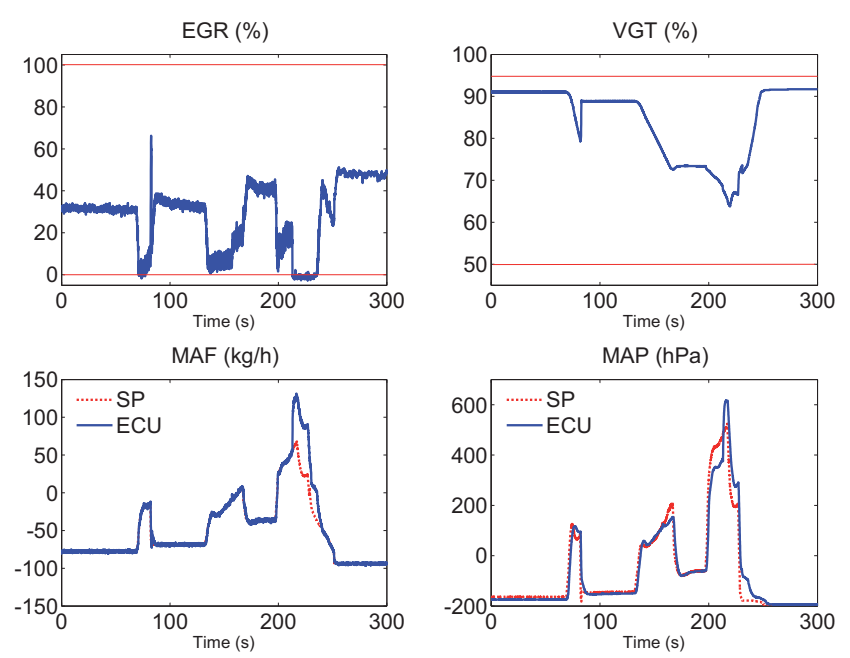

Figure 15

Experimental results concerning the ECU under the extraurban part of the NEDC. Despite of a good tracking performance in the first part of the scenario, especially for the MAF, the tracking performance deteriorates on the high speed parts of the cycle.

NEDC. Figure 15 shows the ECU's tracking performance. The MAF is correctly tracked until $t=210$, where the engine speed exceeds the 2100 RPM (Fig. 11). The same deterioration at high speed is also noted on the MAP tracking performance. On the other hand, the NMPC approach 

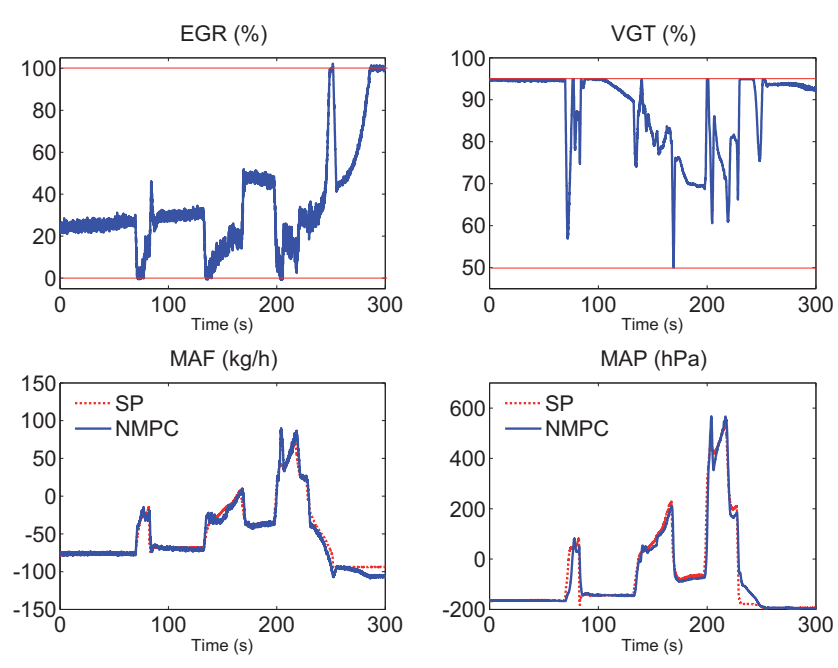

Figure 16

Experimental results using the proposed parameterized NMPC scheme with the standard parameters depicted in Table 1. Note that both variables are correctly tracked, especially in the highspeed part.

presented in Figure 16 clearly shows a more regular quality in the tracking performance for both variables comparing to the ECU's, except in the last part at low speed and fuel, where model uncertainties become too high to compensate. Moreover, the slow dynamics of the engine speed profile of the NEDC does not generate abrupt set-point variations, and naturally filters oscillations and overshoots.

However, the present solution seems to be quite sensitive to the fast set-point variations. This is probably due to the fact that the updating rate is not fast enough to compensate for model uncertainties. The computation time that is needed to perform the computation is beyond the $10 \mathrm{~ms}$ which means that a model with lower sampling period can still be used with certainly best performance in terms of tracking. Consequently, one may suggest that the current performance can be viewed as a pessimistic estimation of the best achievable performance with a high sampling rate model.

\section{CONCLUSION AND FUTURE WORKS}

In this paper, an experimental validation of an output feedback based on a NMPC scheme was presented for a diesel engine air path. First results show that the parameterized NMPC solution together with a MHO are real-time implementable while constraints on the inputs are structurally handled. The tracking performance is quite promising when compared to the existing controller.
Nevertheless, the main contribution of the paper is the fact that the present control scheme can work as a blackbox solution for diesel engines in general, dealing with more sophisticated and faster models. The success of this NMPC strategy on such a structure-free model would emphasize our claim on the generic feature of the proposed approach and its complete independence with respect to any structural assumptions.

First simulation results using the proposed controller on a fully sixth-order non linear model are quite promising and will be reported in future communications.

\section{ACKNOWLEDGMENTS}

The sponsoring of this work by the COMET K2 Center "Austrian Center of Competence in Mechatronics (ACCM)" is gratefully acknowledged. The COMET Program is funded by the Austrian Federal Government, the Federal State Upper Austria and the Scientific Partners of ACCM.

\section{References}

Alamir M. (1999) Optimization based non-linear observers revisited. Int. J. Control 72, 13, 1204-1217.

Alamir M. (2006) Stabilization of Nonlinear System Using Receding-Horizon Control Schemes: A parametrized approach for Fast Systems, Lecture Notes in Control and Information Science, Springer, London, ISBN 1-84628470-8.

Alamir M. (2009) A Framework for Monitoring Control Updating Period in Real-Time NMPC, in: Magni L., Raimondo D., Allgöwer F. (eds.), Nonlinear Model Predictive Control, Lecture Notes in Control and Information Science, Springer-Verlag, Heidelberg, Vol. 384, pp. 433-445.

Alamir M., Calvillo-Corona L. (2002) Further results on nonlinear receding horizon observers, IEEE Trans. Automat. Contr. 47, 7, 1188-1193.

Bemporad A., Morari M., Dua V., Pistikopoulos E.N. (2002) The explicit linear quadratic regulator for constrained systems, Automatica 38, 3-20.

Chauvin J., Corde G., Petit N., Rouchon P. (2008) Motion planning for experimental airpath control of a diesel homogeneous charge-compression ignition engine, Control Eng. Pract. 16, 1081-1091.

Christen U., Vantine K.J., Collings N. (2001) Event-based mean-value modeling of DI Diesel engines for controller design, SAE paper 2001-01-1242.

Diehl M., Bock H.G., Schloöder J.P. (2005) A real-time iteration scheme for nonlinear optimization in optimal feedback control, Siam J. Control Optim. 43, 1714-1736. 
Ferreau H.J., Bock H.G., Diehl M. (2006) An online active set strategy for fast parametric quadratic programming in MPC applications, Proceedings of the IFAC Workshop on Nonlinear Model Predictive Control for Fast Systems, Grenoble.

Ferreau H.J., Ortner P., Langthaler P., del Re L., Diehl M. (2007) Predictive control of a real-world Diesel engine using an extended online active set strategy, Annu. Rev. Control 31, 293-301.

Fliess M., Lévine J., Martin P., Rouchon P. (1992) On differentially flat nonlinear systems, IFAC-Symp. NOLCOS'92, Bordeaux, pp. 408-412.

Guzzella L., Onder C. (2010) Introduction to Modeling and Control of Internal Combustion Engine Systems, Springer-Verlag, Berlin, 2nd edition.

Heywood J.B. (1998) Internal combustion engine fundamentals, McGraw-Hill.

Jacobs T., Assanis D., Filipi Z. (2003) The impact of exhaust gas recirculation on performance and emissions of a heavy-duty diesel engine, SAE paper 2003-01-1068.

Jankovic M., Kolmanovsky I. (1998) Robust nonlinear controller for turbocharged Diesel engines, Proceedings of the American Control Conference, Philadelphia.

Johnson T.V. (2001) Diesel emission control in review, $S A E$ paper 2001-01-0184.

Jung M. (2003) Mean-value modelling and robust control of the airpath of a turbocharged Diesel engine, PhD Dissertation, University of Cambridge.

Mayne D.Q., Rawlings J.B., Rao C.V., Scokaert P.O.M. (2000) Constrained model predictive control: Stability and optimality, Automatica 36, 6, 789-814.

Michalska H., Mayne D.Q. (1995) Moving horizon observers and observer based control, IEEE Trans. Automat. Control 40, 6, 995-1006.

Murilo A., Alamir M., Ortner P. (2009) Fast NMPC Scheme for a Diesel Engine Air Path, Proceedings of the European Control Conference (ECC'09), Budapest.
Nieuwstadt M.J.V., Moraal P.E., Kolmanovsky I.V., Stefanopoulou A., Wood, P., Criddle M. (1998) Decentralized and multivariable designs for EGR-VGT control of a Diesel engine, IFAC Workshop on Advances in Automotive Control, Mohican State Park, $\mathrm{OH}$.

Ohtsuka T. (2004) A continuation/gmres method for fast computation of nonlinear receding-horizon control, Automatica 40, 563-574.

Ortner P., Bergmann R., Ferreau H.J., del Re L. (2009) Nonlinear Model Predictive Control of a Diesel Engine Airpath, IFAC Workshop on Control Applications of Optimisation, Jyvaskyla.

Ortner P., del Re L. (2007) Predictive control of a Diesel engine air path, IEEE Trans. Control Syst. Technol. 15, 3 , 449-456.

Plianos A., Stobart R. (2007) Dynamic feedback linearization of Diesel engines with intake variable valve actuation, IEEE International Conference on Control Applications, Singapore, pp. 455-460.

van Nieuwstadt M.J., Kolmanovsky I.V., Moraal P.E. (2000) Coordinated EGR-VGT control for Diesel engines: An experimental comparison, SAE paper 2000-01-0266.

Wei X. (2006) Advanced LPV techniques for Diesel engines, PhD Dissertation, Johannes Kepler University, Grenoble.

Zavala V.M., Laird C.D., Biegler L.T. (2006) Fast solvers and rigorous models: can both be accommodated in NMPC? Proceedings of the IFAC Workshop on Nonlinear Model Predictive Control for Fast Systems.

Final manuscript received in March 2011 Published online in September 2011 